\title{
The Need for a Better-Designed Study of the Outcomes of Endoscopic Management of Bile Leak
}

\author{
Hyung Ku Chon ${ }^{1,2}$, Eun Ji Shin ${ }^{3}$ and Seong-Hun Kim ${ }^{4}$ \\ ${ }^{1}$ Department of Internal Medicine, ${ }^{2}$ Institute of Wonkwang Medical Science, Wonkwang University College of Medicine, Iksan, Korea, \\ ${ }^{3}$ Division of Gastroenterology and Hepatology, Johns Hopkins University, Baltimore, MD, USA, ${ }^{4}$ Department of Internal Medicine, \\ Research Institute of Clinical Medicine of Jeonbuk National University-Biomedical Research Institute of Jeonbuk National University \\ Hospital, Jeonju, Korea
}

See "Outcomes of Endoscopic Management among Patients with Bile Leak of Various Etiologies at a Tertiary Care Center" by Suprabhat Giri, Sridhar Sundaram, Harish Darak, et al., on page 727-734.

Bile leak occurs commonly after surgery, particularly following cholecystectomy, liver transplantation, partial liver resection, or hydatid cyst excision. ${ }^{1,2}$ Non-surgical bile leak can occur in association with liver abscess or trauma. ${ }^{3}$ Early recognition and diagnosis of bile leak are important because untreated ductal disruptions can result in significant morbidity and mortality. The treatment of bile leak requires a multidisciplinary approach, including surgery, radiology, and interventional endoscopy. ${ }^{4} \mathrm{~A}$ variety of percutaneous, endoscopic, and surgical methods have been proposed for the management of bile leak. A percutaneous approach may be preferred in cases of a large fluid collection resulting from bile leak. Complete transection of the bile duct or bile leak refractory to endoscopic or percutaneous management may require an additional surgery. However, endoscopic retrograde cholangiopancreatography (ERCP) with biliary sphincterotomy, biliary stenting, nasobiliary tube insertion, or with a combination of these techniques is considered the first-line treatment modality.

Received: September 30, 2020 Revised: October 10, 2020

Accepted: October 13, 2020

Correspondence: Seong-Hun Kim

Department of Internal Medicine, Research Institute of Clinical Medicine of Jeonbuk National University-Biomedical Research Institute of Jeonbuk National University Hospital, 20 Geonji-ro, Deokjin-gu, Jeonju 54907, Korea Tel: +82-63-250-2295, Fax: +82-63-254-1609, E-mail: shkimgi@jbnu.ac.kr ORCID: https://orcid.org/0000-0002-7592-8060

(cc) This is an Open Access article distributed under the terms of the Creative Commons Attribution Non-Commercial License (http://creativecommons.org/ licenses/by-nc/3.0) which permits unrestricted non-commercial use, distribution, and reproduction in any medium, provided the original work is properly cited.
Early healing of ductal disruption may be achieved using these endoscopic procedures as they reduce the transpapillary pressure gradient between the bile duct and duodenum, facilitating bile flow into the duodenum rather than into the leakage site. ${ }^{5}$ A systematic review has reported the success rate of endoscopic therapy for bile leak to be $68 \%$ to $98.3 \% .^{6}$ The current proposed treatment algorithm for bile leak is shown in Fig. $1 .^{4}$

In a study published in this issue of Clinical Endoscopy, Giri et al. ${ }^{3}$ retrospectively evaluated the efficacy and outcomes of endoscopic treatment for bile leak of various etiologies including cholecystectomy $(n=34)$, liver abscess $(n=20)$, hydatid cyst surgery $(n=11)$, other surgeries $(n=5)$, and trauma $(n=1)$.

All patients enrolled in this study were intended to undergo biliary sphincterotomy with leak-bridging biliary stenting via ERCP; however, one patient with complete transection of the common bile duct underwent surgery after biliary sphincterotomy. The primary outcome of complete symptomatic resolution without the leakage of contrast material during the second ERCP performed 6 weeks after the initial ERCP was achieved in $91.5 \%$ patients. Procedural adverse events were post-ERCP pancreatitis $(n=4,5.6 \%)$ and post-endoscopic sphincterotomy bleeding $(n=1,1.4 \%)$.

This study is limited by its retrospective nature and small sample size. Interestingly, no differences in outcomes due to the timing of ERCP, size of the stent used, and etiology of bile leak (surgical and non-surgical groups) were reported. Nevertheless, caution should be taken when interpreting these findings. 


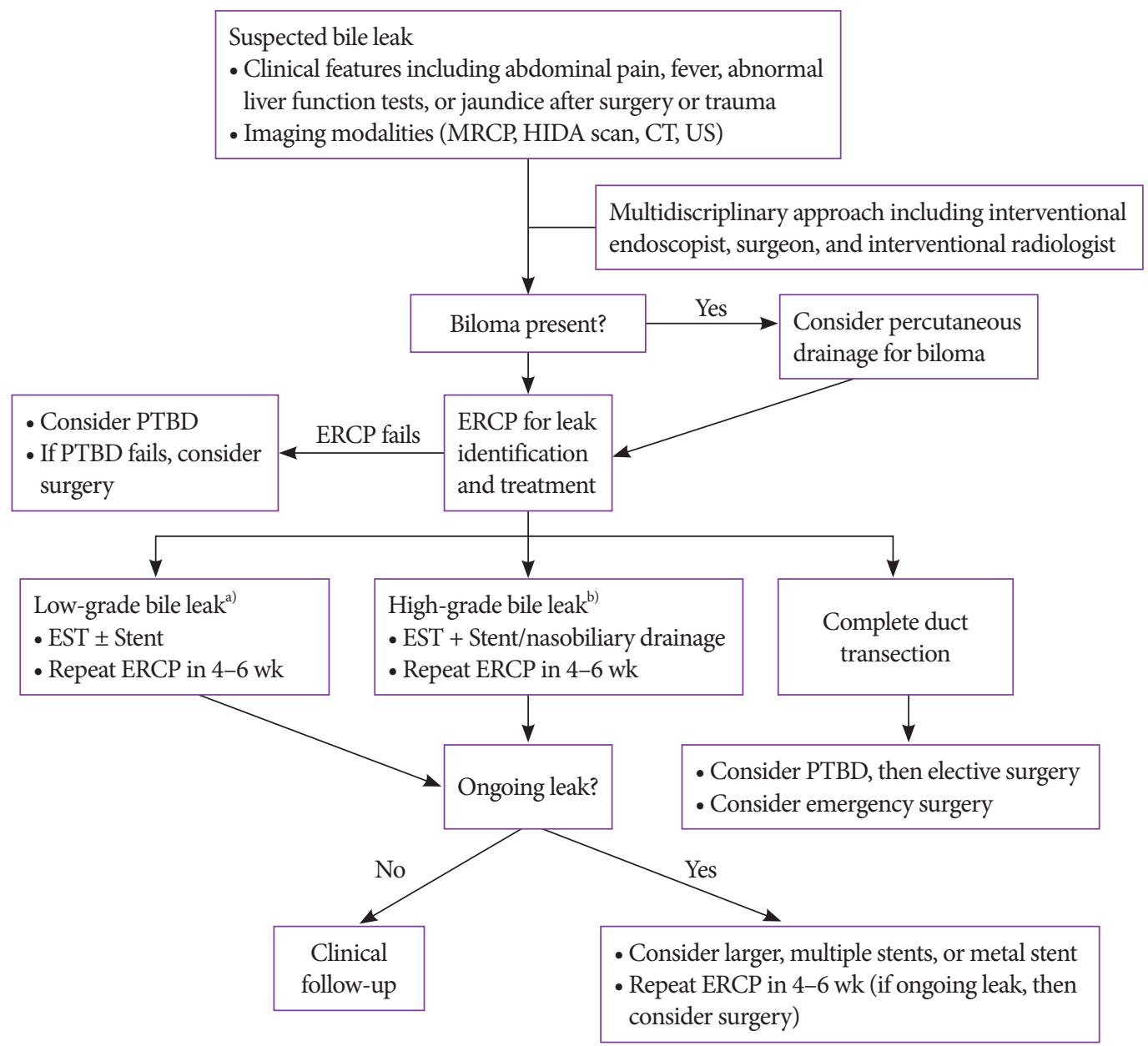

Fig. 1. Suggested treatment algorithm for bile leak. CT, computed tomography; ERCP, endoscopic retrograde cholangiopancreatography; EST, endoscopic sphincteroromy; HIDA, hepatobiliary iminodiacetic acid; MRCP, magenetic resonance cholangiopancreatography; PTBD, percutaneous transhepatic biliary drainage; US,

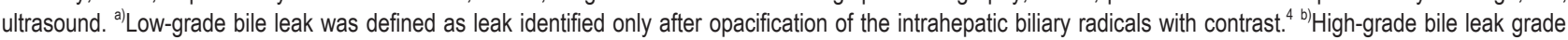
was defined as leak observed fluoroscopically before intrahepatic opacification. ${ }^{4}$

Regarding the timing of ERCP, detailed information of patients who underwent ERCP after 1 day, within 2-3 days, and after 3 days of diagnosis were not available, and there was a lack of explanation for the delay in performing ERCP. Subhepatic drainage tube insertion after cholecystectomy or percutaneous drainage of localized fluid collections before ERCP, as mentioned in the treatment protocol, might affect the result. However, there was no relation between the timing of ERCP and clinical outcomes.

Theoretically, a large-diameter plastic stent can enhance bile flow compared to one with a small-diameter. In this study, the authors did not describe the severity of bile leak in all patients. In cases of a major bile leak, a small-diameter plastic stent may be ineffective. In a systematic review, comparison of small- and large-diameter stents showed no significant difference in clinical success. ${ }^{5}$ However, the review mostly included cases of minor bile leaks, making it difficult to generalize the findings to the treatment of major bile leaks.

In this study, there was no significant difference in outcomes between the surgical and non-surgical groups except the length of hospitalization. This is an interesting finding that has not been reported before; however, the reason behind this finding remains unknown.

This study demonstrated that biliary stent placement via ERCP is important for the management of bile leakage of various etiologies despite the delay in performing ERCP. However, additional well-designed large prospective cohort studies are needed to validate the results of this study. 
Conflicts of Interest

The authors have no financial conflicts of interest.

ORCID

Hyung Ku Chon: https://orcid.org/0000-0002-6068-3849

Eun Ji Shin: https://orcid.org/0000-0003-0624-8149

\section{REFERENCES}

1. Kim KH, Kim TN. Endoscopic management of bile leakage after cholecystectomy: a single-center experience for 12 years. Clin Endosc 2014;47:248-253.

2. Raza A, Omer A, Iqbal S, Gudsoorkar V, Koduru P, Krishnan K. Efficacy of nasobiliary tubes and biliary stents in management of patients with bile leak after liver transplantation: a systematic review. Clin Endosc 2019;52:159-167.

3. Giri S, Sundaram S, Darak H, Kumar S, Bhatia S. Outcomes of endoscopic management among patients with bile leak of various etiologies at a tertiary care center. Clin Endosc 2020;53:727-734.

4. Sandha GS, Bourke MJ, Haber GB, Kortan PP. Endoscopic therapy for bile leak based on a new classification: results in 207 patients. Gastrointest Endosc 2004;60:567-574.

5. Bjorkman DJ, Carr-Locke DL, Lichtenstein DR, et al. Postsurgical bile leaks: endoscopic obliteration of the transpapillary pressure gradient is enough. Am J Gastroenterol 1995;90:2128-2133.

6. Vlaemynck K, Lahousse L, Vanlander A, Piessevaux H, Hindryckx P. Endoscopic management of biliary leaks: a systematic review with meta-analysis. Endoscopy 2019;51:1074-1081. 